\title{
Hybrid ZnO Flowers-Rods Nanostructure for Improved Photodetection Compared to Standalone Flowers and Rods
}

\author{
Abdullah M. Al-Enizi ${ }^{1, *}\left(\mathbb{D}\right.$, Shoyebmohamad F. Shaikh ${ }^{1}{ }^{\circledR}$, Asiya M. Tamboli ${ }^{2, *}$, Afifa Marium ${ }^{3}$, \\ Muhammad Fazal Ijaz ${ }^{3}{ }^{(D}$, Mohd Ubaidullah $^{1}\left(\mathbb{D}\right.$, Meera Moydeen Abdulhameed $^{1}$ (D) and Satish U. Ekar ${ }^{4, *}$ \\ 1 Department of Chemistry, College of Science, King Saud University, Riyadh 11451, Saudi Arabia; \\ sshaikh1@ksu.edu.sa (S.F.S.); mtayyab@ksu.edu.sa (M.U.); meeranis@yahoo.co.in (M.M.A.) \\ 2 School of Chemical Engineering, Yeungnam University, 280 Daehak-Ro, Gyeongsan 38541, Korea \\ 3 Department of Intelligent Mechatronics Engineering, Sejong University, Seoul 05006, Korea; \\ mariumafifa1440@gmail.com (A.M.); fazal@sejong.ac.kr (M.F.I.) \\ 4 Department of Physics, Baburaoji Gholap College, Pune 411027, Maharashtra, India \\ * Correspondence: amenizi@ksu.edu.sa (A.M.A.-E.); asiyashaikh2020@gmail.com (A.M.T.); \\ satishuekar@gmail.com (S.U.E.)
}

check for updates

Citation: Al-Enizi, A.M.; Shaikh, S.F.; Tamboli, A.M.; Marium, A.; Ijaz, M.F.; Ubaidullah, M.; Moydeen

Abdulhameed, M.; Ekar, S.U. Hybrid ZnO Flowers-Rods Nanostructure for Improved Photodetection Compared to Standalone Flowers and Rods. Coatings 2021, 11, 1464. https:// doi.org/10.3390/coatings11121464

Academic Editor: Aivaras Kareiva

Received: 27 October 2021

Accepted: 21 November 2021

Published: 29 November 2021

Publisher's Note: MDPI stays neutral with regard to jurisdictional claims in published maps and institutional affiliations.

Copyright: (c) 2021 by the authors. Licensee MDPI, Basel, Switzerland. This article is an open access article distributed under the terms and conditions of the Creative Commons Attribution (CC BY) license (https:/ / creativecommons.org/licenses/by/ $4.0 /)$.

\begin{abstract}
Different Zinc Oxide ( $\mathrm{ZnO})$ morphologies have been used to improve photodetector efficiencies for optoelectronic applications. Herein, we present the very novel hybrid ZnO flower-rod (HZFR) morphology, to improve photodetector response and efficiency when compared to the prevalently used $\mathrm{ZnO}$ nanorods (NRs) and $\mathrm{ZnO}$ nanoflowers (NFs). The HZFR was fabricated via sol-gel microwave-assisted hydrothermal methods. HZFR achieves the benefits of both NFs, by trapping a greater amount of UV light for the generation of e-h pairs, and NRs, by effectively transporting the generated e-h pairs to the channel. The fabricated photosensors were characterized with scanning electron microscopy, X-ray diffraction, photoluminescence, and a Keithley 4200A-SCS parameter analyzer for their morphology, structural characteristics, optical performance, and electrical characteristics, respectively. The transient current response, current-voltage characteristics, and responsivity measurements were set as a benchmark of success to compare the sensor response of the three different morphologies. It was found that the novel HZFR showed the best UV sensor performance with the fastest response time ( 7 s), the highest on-off ratio (52), and the best responsivity (126 A/W) when compared to the NRs and NFs. Hence, it was inferred that the HZFR morphology would be a great addition to the $\mathrm{ZnO}$ family for photodetector applications.
\end{abstract}

Keywords: ZnO; UV sensors; photodetectors; nanorods; nanoflowers; hybrid ZnO flower-rod; responsivity; response time

\section{Introduction}

In the contemporary science of photonics, $\mathrm{ZnO}$ is in high demand to replace silicon technology for optoelectronics [1-4]. ZnO is an amazing multifunctional material, which strategically lies on the border of ionic and covalent compounds and has potential applications in biomedicine, environmental monitoring, healthcare, photonics, optoelectronics, and advanced manufacturing. It is because of its room temperature, high-exciton binding energy (60 meV), and, most importantly, its large and direct bandgap of $3.37 \mathrm{eV}$, which makes it more pragmatic in optoelectronics. Furthermore, the possibility of fabricating multiple polymorphic shapes of $\mathrm{ZnO}$, including nanowires, nanoflowers, nanoparticles, nanorods, nanowalls, nanotubes, nanotetrapods, quantum dots, and more, has made it even more relevant in the realms of optoelectronics, photonics, and nanoelectronics [5-8]. There is plenty of research with a focus on fabricating novel $\mathrm{ZnO}$ nanoscale morphologies and shapes, to improve the surface-area-to-volume ratio for optoelectronic devices [9]. Hence, for the first time, we fabricated the nanoscale hybrid $\mathrm{ZnO}$ flower-rod (HZFR) morphology for use in optoelectronics, photonics, nanoelectronics, and sensors technology. 
In this study, the novel HZFR was used to obtain the best results for $\mathrm{ZnO}$ ultraviolet (UV) sensors and photodetectors when compared to different traditional and prevalent morphologies, including $\mathrm{ZnO}$ nanorods (NRs) and $\mathrm{ZnO}$ nanoflowers (NFs).

$\mathrm{ZnO}$ strategically lies at the borderline of inorganic and organic chemical compounds, which makes it a very important compound for sensor applications, due to its intertwined chemical and optical properties [10,11]. Notwithstanding, the more pronounced surface oxygen chemisorption process at ambient conditions makes it one of the best candidates for UV and gas sensor applications [12,13]. Moreover, the visible blind intrinsic habit and very high absorption in the UV band provide a great impetus to ZnO-based UV sensors [14]. Researchers exploit multiple techniques to achieve a fast impulse response time, a high light-to-dark (on-off) current ratio, and a high responsivity and photoconductive gain in $\mathrm{ZnO}$-based photosensors. Some of the most prevalent techniques are to change the morphology for an improved surface-to-volume ratio, as well as surface functionalization with highly light-absorbing materials, stimulating the surface Schottky barriers via metal nanoparticles decoration, channel doping, and improving the overall $\mathrm{ZnO}$ crystal quality for intrinsic defects engineering [15-19]. However, the most pronounced, established, and easy-going approach is morphology-oriented; a change in morphology leads to an improved surface-to-volume ratio for best UV sensor efficiency.

For all the aforementioned purposes, $\mathrm{ZnO}$ has been fabricated with different solutionprocessed and vacuum-based methods, including molecular beam epitaxy, thermal evaporation, atomic layer deposition (ALD), metal-organic chemical vapor deposition (MOCVD), magnetron sputtering, spin coating, hydrothermal processes, solvothermal growth, and microwave-assisted growth (MAG) methods [20-25]. All these methods have their specific pros and cons; however, most vacuum-based methods have the limitation of using very high temperatures, high pressures, costly processes, and limited use of substrates. On the contrary, solution-processed methods have benefits of high nanostructure yield, easy and cheap processing, and handiness. In this study, we fabricated a novel HZFR morphology via the solution-replacement MAG method [25]. The MAG method was chosen based on its unparalleled benefits of ultrafast processing, solution route, facile synthesis, strict control over the process, repeatability, reproducibility, low price, purity, eco-friendly approach, and easy availability on a commercial level [26]. Furthermore, the nanostructures that are fabricated with the MAG methods have a high crystal quality with the least defects, which are the basic requirements for better UV sensor response [19].

The prime focus of this study was neither stimulating the nanostructured surface Schottky barrier nor using any surfactants to maximize the UV light absorption. However, we paid attention to the basic ingredients of designing the nanostructured morphology with shapes that had never been used previously to obtain the best results for UV sensors. The presented HZFR morphology exploited the benefits of both NFs and NRs, by trapping as much light as possible for e-h pairs generation and transporting them smoothly to the receiver contacts. Hence, the UV light, that was previously lost between the adjoining NR gaps, was used to produce the maximum gain, best impulse response, highest on-off ratio, and the best responsivity in HZFR. The formed nanostructures were characterized with scanning electron microscopy (SEM), X-ray diffraction (XRD), and photoluminescence (PL) to probe the nanostructured morphology, crystalline structure, and optical characteristics, respectively. The presented results have applications in the fields of photonics, optoelectronics, biomedicine, and nanoscience.

\section{Materials and Methods}

\subsection{Substrate Cleaning}

The first step in the device fabrication was the cleaning of the substrates. For NR, NF, and HZFR film deposition, we used $1.5 \times 1.5 \mathrm{~cm}$ transparent ITO-coated glass substrates. At first, the glass substrates were exposed to $\mathrm{N}_{2}$ blowing, followed by a wet ultrasonic bath in acetone $\left(\mathrm{C}_{3} \mathrm{H}_{6} \mathrm{O},>99.5 \%\right.$, Sigma Aldrich, Riyadh, Saudi Arabia), isopropyl-alcohol $\left(\mathrm{C}_{3} \mathrm{H}_{8} \mathrm{O},>99.5 \%\right.$, Sigma Aldrich), and distilled water. The liquids were evaporated by 
annealing the substrates at $300{ }^{\circ} \mathrm{C}$ for $5 \mathrm{~min}$. In the end, the substrates were again cleaned with $\mathrm{N}_{2}$ blowing, to remove any remaining dirt and dust particles, and were then stored in the cleanroom.

\subsection{Sensors Fabrication with Different Morphologies}

Three different devices were fabricated on the ITO-coated glass substrates with NRs, $\mathrm{NFs}$, and HZFR as the active layers/films. A thin ITO layer was already deposited on the glass substrate and served as a bottom transparent electrode. Then, the active sensor layers, with three different morphologies, were deposited via the following procedures:

\subsubsection{ZnO Nanorods (NRs) Deposition}

The NRs were deposited via the MAG method, as explained by Rana et al. in previous reports [25,26]. The MAG method was chosen due to its ultrafast processing, facile procedures, and high yield in nanostructure fabrication. Herein, we used the microwaveassisted solution replacement method for effective NR deposition. For axial NR growth, the seeds' deposition is inevitable, to support the heterogeneous nucleation process within the solution atop the substrate [27]. Hence, the seed solution was made by mixing zinc acetate dehydrate $\left(\mathrm{Zn}\left(\mathrm{CH}_{3} \mathrm{COO}\right)_{2} \cdot 2 \mathrm{H}_{2} \mathrm{O},>98 \%\right.$, Sigma Aldrich) with n-propanol $\left(\mathrm{C}_{3} \mathrm{H}_{8} \mathrm{O}\right.$, $>99.5 \%$, Sigma Aldrich), to make a $0.05 \mathrm{M}$ solution. The solute and the solvent were ultra-sonicated for $30 \mathrm{~min}$ and the saturated seed solution was secured. The seeds were spin-coated twice on the ITO-coated glass substrate, followed by annealing at $350{ }^{\circ} \mathrm{C}$ for $1 \mathrm{~h}$. Consequently, the growth solution was prepared by stirring $25 \mathrm{mM}$ zinc nitrate hexahydrate $\left(\mathrm{Zn}\left(\mathrm{NO}_{3}\right)_{2} \cdot 6 \mathrm{H}_{2} \mathrm{O},>98 \%\right.$, Sigma Aldrich) and $25 \mathrm{mM}$ hexamethylenetetramine $\left(\mathrm{C}_{6} \mathrm{H}_{12} \mathrm{~N}_{4}, 99 \%\right.$, Sigma Aldrich) with distilled water for $1 \mathrm{~h}$. The total volume of the solution was $300 \mathrm{~mL}$. For growth, a domestic microwave oven was used with a maximum power of 1000 watts, model NN-ST665BQPQ, and manufacturer Panasonic (Macquarie Park, New South Wales, Australia). Then, the seeded substrate was immersed into the homogeneous solution container and exposed to the 1000 watt microwaves, with four intermittent intervals of $30 \mathrm{~s}$ each [25]. After $10 \mathrm{~min}$, and having replaced the fresh stream of solution four times, the substrate was removed from the solution container, cleaned with distilled water, annealed at $300^{\circ} \mathrm{C}$ for $10 \mathrm{~min}$, and dried with $\mathrm{N}_{2}$ blowing. The sample was characterized with SEM to confirm the NR deposition.

\subsubsection{ZnO Nanoflowers (NFs) Deposition}

NFs were also fabricated via the MAG method. The growth solution was prepared by stirring equimolar $(50 \mathrm{mM}) \mathrm{Zn}\left(\mathrm{NO}_{3}\right)_{2} \cdot 6 \mathrm{H}_{2} \mathrm{O}$ and $\mathrm{C}_{6} \mathrm{H}_{12} \mathrm{~N}_{4}$ with distilled water. Then, $10 \mathrm{~mL}$ ammonium hydroxide $\left(\mathrm{NH}_{4} \mathrm{OH}, 28.0-30.0 \% \mathrm{NH}_{3}\right.$ basis) was drop cast into the solution and stirred for $1 \mathrm{~h}$. Afterward, the unseeded ITO-coated glass substrate was immersed into the growth solution and exposed to 1000 watt microwaves with intermittent intervals. After $10 \mathrm{~min}$ and solution replacements, the substrate was removed from the solution and stored in the cleanroom.

\subsubsection{Hybrid ZnO Flower-Rod (HZFR) Deposition}

For HZFR deposition, NRs were initially deposited on the seeded substrate, as explained earlier in Section 2.2.1. In a different container, the NFs were prepared in a solution, as explained in Section 2.2.2, without a substrate. Then, the collected solution containing the NFs was drop-casted onto the substrate with axial NRs and spun at 3000 RPM for $30 \mathrm{~s}$. The spinning process was repeated 10 times. The substrate was annealed at $100{ }^{\circ} \mathrm{C}$ for $2 \mathrm{~min}$ for the first nine coatings, and at $360^{\circ} \mathrm{C}$ for $2 \mathrm{~h}$ for the final, tenth coating, to provide stability and to support the heterogeneous nucleation of the flowers atop the vertical rods. The final fabricated morphology was the HZFR, as confirmed by the SEM images in the Results and Discussion sections. 


\subsection{Final Device Fabrication}

Finally, to produce an Ohmic contact with $\mathrm{ZnO}$, ITO was sputtered atop the nanostructured active layer as a top transparent electrode in all three devices. The sputtering was followed by defining the top and bottom indium (In) dots, for electrical connections with the Keithley workstation probes.

\subsection{Characterization Tools}

The samples were characterized with a scanning electron microscope (SEM: Hitachi S4700, Hitachi, Tokyo, Japan) to probe the physical characteristics and the morphology of the active layer nanostructures. The nanostructures' structural and crystalline properties were measured with X-ray diffraction (XRD: Rigaku D/MAX-2500 V/PC, Tokyo, Japan) analysis. The optical characteristics and the defect sites were measured with photoluminescence (PL: FLS 1000, Livingston, UK) analysis. The transient current response and the electrical characteristics were probed with a Keithley 4200A-SCS parameter analyzer (Keithley Instruments, Solon, OH, USA) for the UV on-off conditions. During the photocurrent measurement, we illuminated the sample with $368 \mathrm{~nm}$ UV light by dispersing a $150 \mathrm{~W}$ deuterium $\left(\mathrm{D}_{2}\right)$ lamp with a monochromator, using a $1 \mathrm{~V}$ applied bias.

\section{Results and Discussion}

\subsection{Novel Nanostructured Morphologies}

Figure 1 shows the SEM morphological analyses of the grown nanostructures via the MAG method. The main Figure 1a shows the equally distributed and vertically wellaligned NRs on the substrate surface. The average height of the NRs was $\sim 2$ microns and the average diameter was $\sim 50 \mathrm{~nm}$. The inset shows the magnified version of the same NRs. It is evident that, even though the NRs are equally distributed and densely populated, there is an obvious space between the adjoining NRs, where the illuminated UV light can be wasted on the substrate. The same is true for the NFs in Figure 1b, where the nanoflowers are haphazardly decorated on the substrate surface. The reason for this lowdensity NF growth is the homogeneous nucleation of the NFs on the substrate surface in the absence of seeds. The seeds were not deposited for the NFs' growth because the buried seeds are 0001-oriented, are vertical to the substrate, and may suppress the formation of $\mathrm{Zn}(\mathrm{OH})_{4}{ }^{2-}$ growth units and $\mathrm{Zn}(\mathrm{OH})_{2}$ nuclei. In the absence of seeds and the presence of $\mathrm{NH}_{4} \mathrm{OH}$-driven. excessive $\mathrm{OH}^{-}$ions, the excessive growth units joined the nuclei via the Ostwald ripening process, and a flower-like structure was formed. Hence, the NFs are not homogeneously decorated on the substrate surface, and left wide spaces between adjoining NFs, as evident in Figure 1b. Despite this, NFs have a high surface-to-volume ratio, due to their spread 3D structure when compared to the 1D structure of NRs, and this spread morphology can trap excessive UV light for a better sensitivity [28]. However, the lowered density might result in the loss of some useful light to the substrate, which may affect the UV sensor efficiency. Figure $1 \mathrm{~b}$ inset shows the magnified image of the single NR with a central nucleus and the attached rod-like petals. Figure 1c shows the fabricated novel HZFR for the improved UV sensor response. The large NFs are grown on the buried NRs to form HZFR. The top NFs are joined by the buried NRs at the active 0001 top surfaces. The inset Figure 1c shows the magnified image of the HZFR, and the main Figure background shows the high density of the NFs on the buried NRs. This novel morphology and orientation might combine the benefits of both NRs and NFs to trap as much UV light as possible, for the best UV sensor results. The UV sensor efficiency was then confirmed via the experiments described in the following sections of the manuscript. 

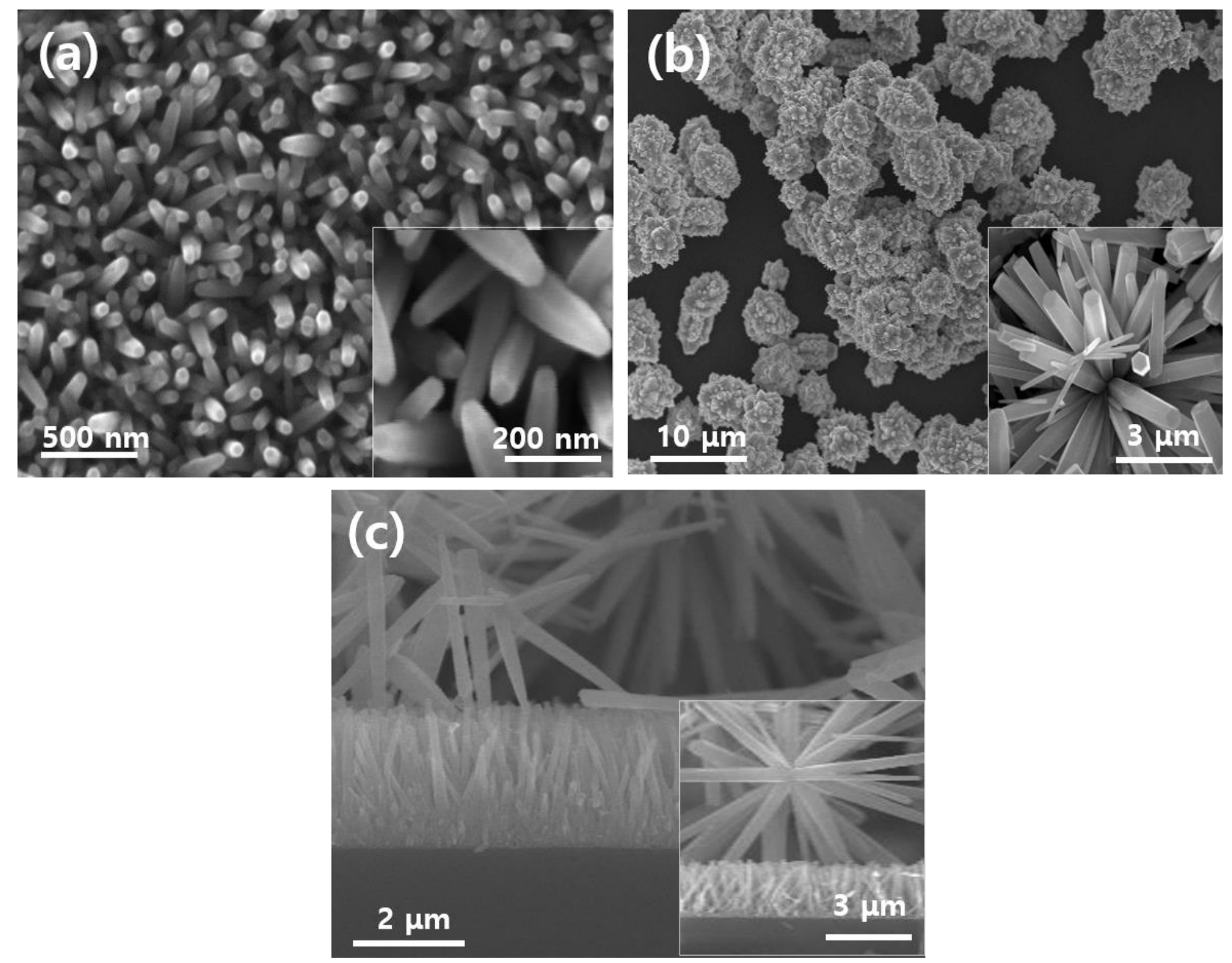

Figure 1. Real-time SEM images of (a) NRs; (b) NFs and (c) HZFR.

\subsection{Nanostructured XRD Structural and PL Optical Characteristics}

The XRD analyses were used to realize the overall crystalline structure and the chemical habits of the grown materials. Figure 2 shows the crystalline patterns of the NRs, NFs, and HZFR XRD analyses. It is evident that all three morphologies are purely made of polycrystalline $\mathrm{ZnO}$, and there is no trace of any foreign elements in the crystalline structures. The grown nanostructures had a wurtzite hexagonal phase, and all the peaks were perfectly matched with the JCPDS number 01-070-8072, which is reported in Figure 2d. The highest peak for the NRs is along the (002) direction (Figure 2a), which is followed by the SEM analyses. On the contrary, the dominant peaks for the NFs are (100) and (101), which are again in line with the SEM images, and confirm the non-vertical growth orientation of NFs on the substrate surface. Similarly, due to the combination of the NRs and NFs, the first dominant peak for HZFR is (101), because of the top NFs, and the second dominant peak is (002), because of the buried NRs beneath the NFs in HZFR. Although the HZFR has vertical rods that are buried beneath the flowers, the XRD orientation is due to the highly magnified and densely populated top flowers that dominate the crystal habit. Table 1 shows the detailed structural parameters of the nanostructures. The full width of half maximum (FWHM) measurements were taken from the (002) peaks, and the crystallite size was measured via the Scherrer formula $[29,30]$.

$$
\mathrm{D}=(0.89 \lambda) /(B \cos \theta)
$$



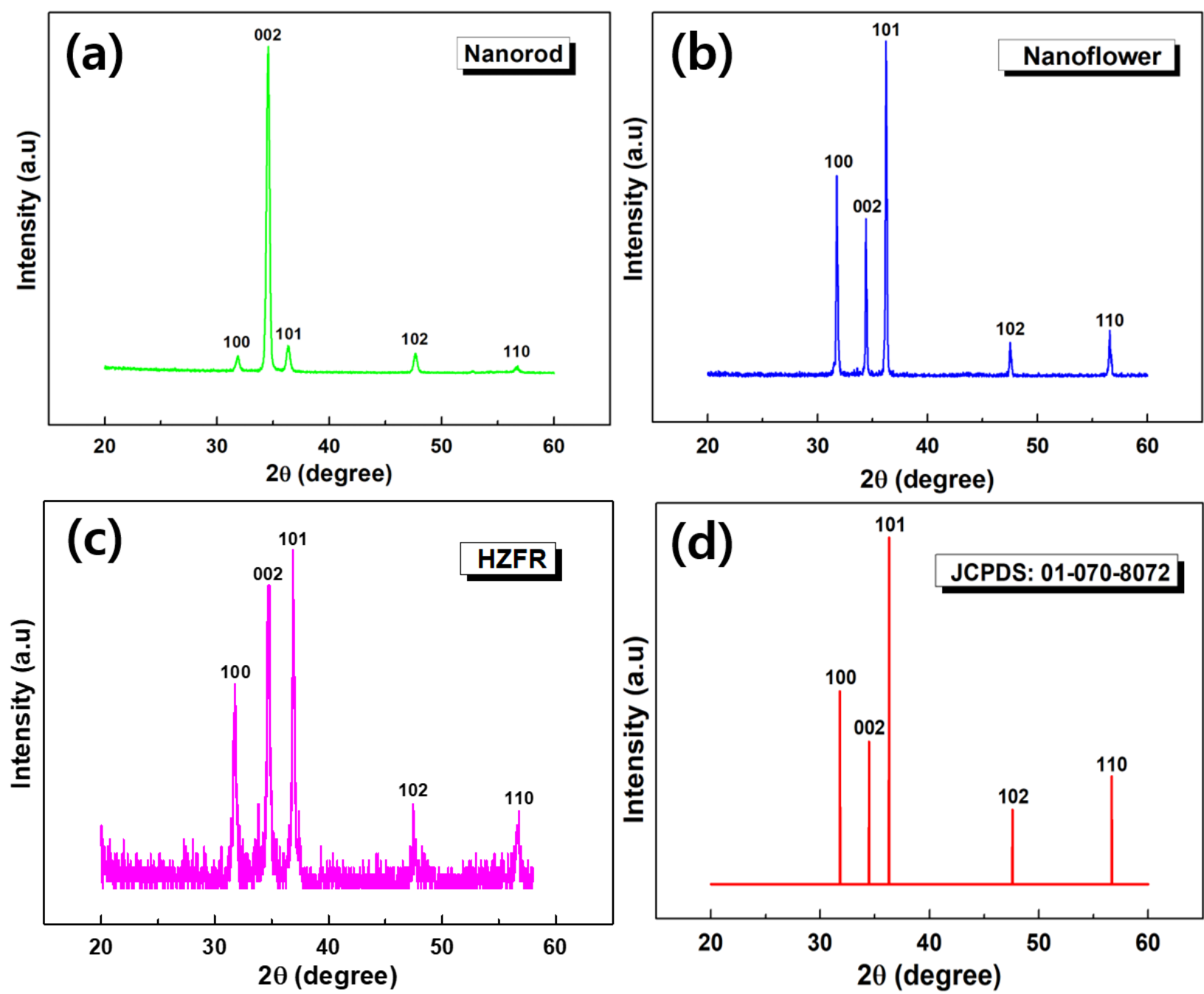

Figure 2. XRD crystalline analyses of (a) NRs; (b) NFs; (c) HZFR and (d) ZnO JCPDS reference.

Table 1. Structural parameters of S1, S2, and S3.

\begin{tabular}{cccc}
\hline Sample & $\begin{array}{c}\text { Two Theta } \\
\text { (Degree) }\end{array}$ & $\begin{array}{c}\text { Full Width at Half } \\
\text { Maximum } \\
\text { (Radian) }\end{array}$ & $\begin{array}{c}\text { Crystallite Size } \\
\text { (nm) }\end{array}$ \\
\hline NRs & 34.43 & 0.0026 & 67 \\
NFs & 34.60 & 0.0031 & 51 \\
HZFRs & 34.78 & 0.0023 & 66 \\
\hline
\end{tabular}

One notable point to ponder on is that the crystallite size of all the nanostructures had values that were comparable to the nanostructures that were fabricated via more sophisticated and expensive methods, such as MOCVD and ALD. This is due to the very effective heating process in the microwave oven that allowed all the atoms to sit on the proper structural sites in the crystal lattice via the dipolar polarization process [25,31]. This is another reason that the MAG nanostructures have better UV sensor efficiencies than other commercially available deposition methods that are available in the market for laboratory testing [19].

Figure 3a shows the room temperature PL optical characteristics of the nanostructures. All the nanostructures, NRs, NFs, and HZFR, show the typical two peaks in the UV and the visible regions for $\mathrm{ZnO}$. The high-intensity peaks in the UV region are due to the exciton recombination process. On the contrary, the low-intensity broad peaks in the visible region are due to the defects that were induced in the materials' crystal structures during the 
growth process. Typical to $\mathrm{ZnO}$, these defects include the $\mathrm{Zn}$ interstitials and the oxygen vacancies [32]. It is noteworthy that all three samples had more or less similar optical profiles, except for a slight redshift in the HZFR PL UV band peak. This redshift was because of the bandgap renormalization effects, the very large dimensions, and the lattice stress that was imbibed during the growth process of HZFR [33,34]. It is obvious that the HZFR is an amalgam of NRs and NFs; hence, HZFR would have excessive free carriers in the channel when compared to NRs and NFs alone. The bandgap renormalization was the direct effect of this excessive carrier-induced, free-carrier screenings and many-body effects present in HZFR structures when compared to the NRs and NFs. Furthermore, the very high dimensions of the NFs atop NRs are a secondary reason for this redshift. However, it is because of the MAG process that, despite different shapes, the inside chemistry and the growth dynamics remained the same, which led to the controlled defect structures in all three samples and resulted in similar peak intensities in the visible region. This would be very helpful to compare the UV sensor responses of all the researched nanostructures, solely based on the change in morphology rather than any other internal factors, such as the lattice parameters and surface- or deep-level defects. Figure $3 b$ shows the UV-Vis diffuse reflectance spectra of $\mathrm{ZnO} N$ Rs, NFs, and HZFR. The different morphologies of $\mathrm{ZnO}$ demonstrate a strong absorption at a wavelength in the range of $250-400 \mathrm{~nm}$. The optical band gap $\mathrm{E}_{\mathrm{g}}$ of the $\mathrm{ZnO}$ samples was estimated by the extrapolation of the linear portion of the plots of $(\mathrm{Ahv})^{2}$ versus hv, i.e., $\mathrm{ZnO}$ NRs $(3.25 \mathrm{eV}), \mathrm{NFs}(3.24 \mathrm{eV})$, and HZFR $(3.23 \mathrm{eV})$.
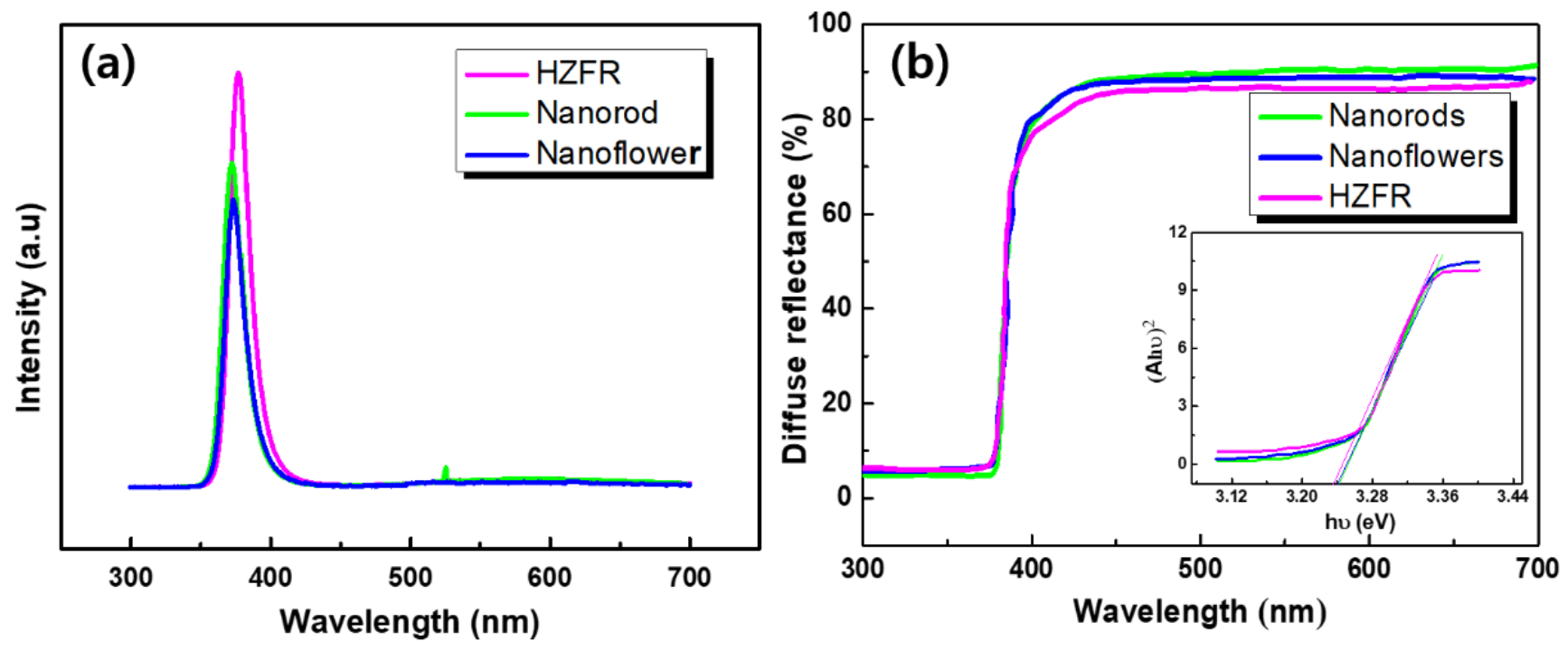

Figure 3. (a) Optical (PL) characteristics of the nanostructures and (b) Uv-Vis diffuse reflectance spectra; the inset is the plot of $(\mathrm{Ahv})^{2}$ versus hv demonstrating the direct bandgap of $\mathrm{ZnO}$.

\subsection{Electrical (UV On-Off) Characteristics and Impulse Response}

Figure 4 shows the electrical current-voltage $(I-V)$ characteristics that were measured via a Keithley 4200A-SCS parameter analyzer for UV on-off conditions. The UV illumination wavelength $\left(\lambda_{\max }\right)$ for the sensor response was found by the equation

$$
\lambda_{\max } \leq h c / E_{g} \approx 368
$$

where $c$ is the speed of light, $h$ is Planck's constant, and $E_{g}$ is the $\mathrm{ZnO}$ bandgap energy. Hence, we set the UV illumination wavelength to $368 \mathrm{~nm}$ for the $I-V$ experimental analyses. 

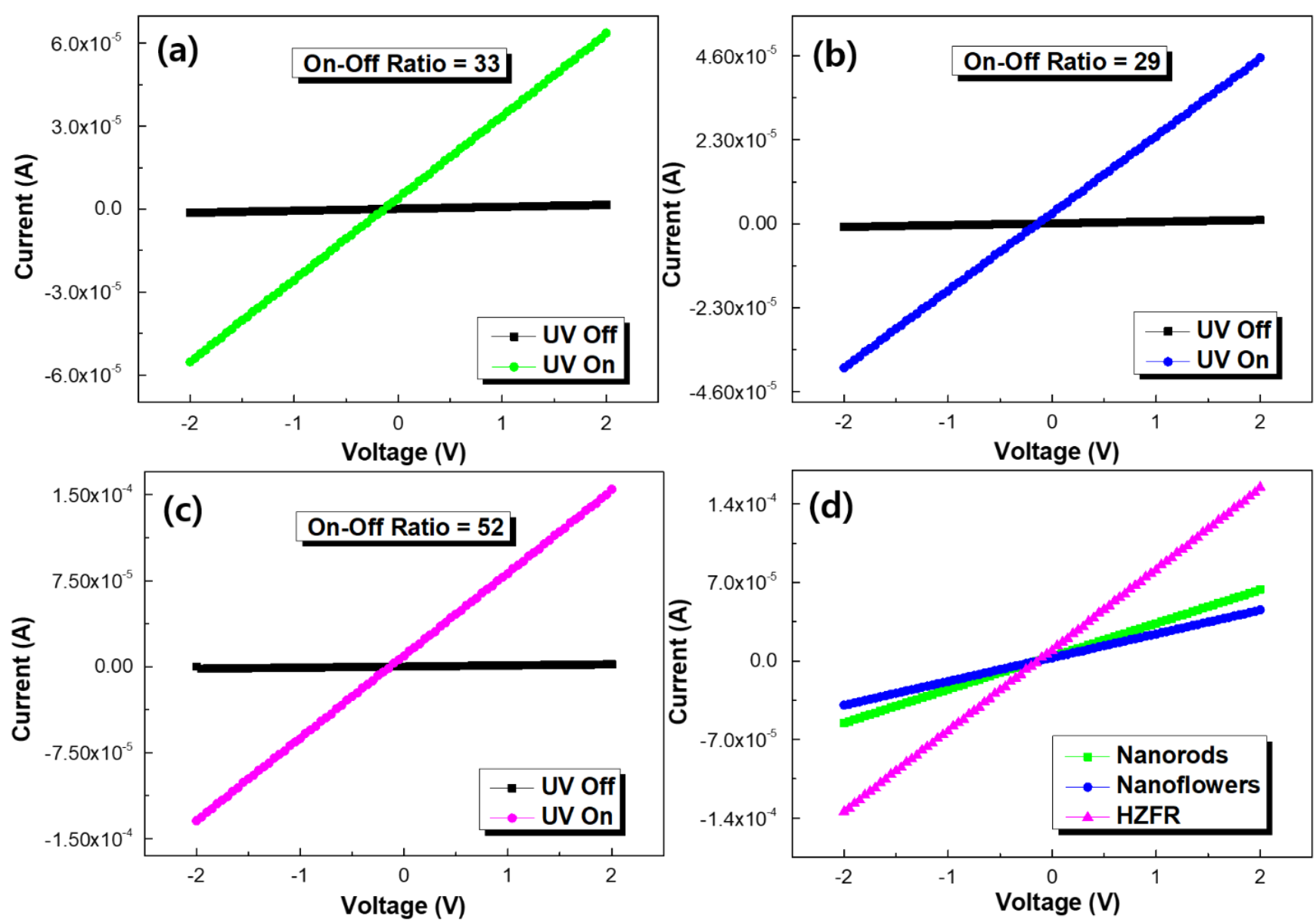

Figure 4. The current-voltage ( $I-V$ ) characteristics of (a) NRs; (b) NFs; (c) HZFR and (d) comparative analysis of $I-V$ characteristics of NRs, NFs, and HZFR under $368 \mathrm{~nm}$ UV illumination.

The total current density is the sum of the electronic and the hole current densities $\left(J=J_{e}+J_{h}\right)$; however, we were only interested in the electronic current density, because $\mathrm{ZnO}$ is intrinsically an n-type semiconductor. Figure 4 shows the current response of the sensors from the -2 to $2 \mathrm{~V}$ sweep. Each Figure shows a dark current behavior (black curves) and a UV-illuminated current (colored curves). The ratio of the maximum illuminated current density to the maximum dark current density is known as the sensor on-off ratio, and this has significant importance in research and development. It defines the extent to which the sensor is sensitive to the applied bias for switching or signaling. It is the on-off ratio that decides the fate of the sensor being used in sophisticated applications, such as wearable devices, biosensors, and optical biopsies. It is evident in Figure $4 a-c$ that all the sensors had almost the same dark current intensities. This was due to the controlled defects' structures within the crystal, via the MAG method in all samples. Nonetheless, all samples show a significant increase in current after the UV illumination. However, the best on-off ratio of ' 52 ' was recorded by the HZFR (Figure 4c) sample, due to its very high surface-to-volume ratio and its trapping of as much UV light as possible to generate e-h pairs. Although the NFs had a high surface-to-volume ratio when compared to NRs, the NFs lack a well-defined electron pathway when compared to the NRs, and therefore display less current at the same voltage. Figure $4 \mathrm{~d}$ shows the comparative analysis of the three sensors for the UV on-off conditions, which further supported the gigantic increase in the HZFR illuminated current intensity when compared to the NRs and NFs. Additionally, the straight-line graphs testify to a perfect Ohmic contact that was formed between $\mathrm{ZnO}$ and ITO/Ag. 
Figure 5 shows the impulse response as a function of time in seconds for UV on-off conditions, at a constant voltage supply of $2 \mathrm{~V}$. All the samples depicted a typical sinusoidal response to the UV on-off conditions. The dark and illuminated current values matched well with the corresponding current intensities in Figure 4. All samples had the three distinct regions of rising (response), saturation, and fall (recovery). The transient current response is an important parameter that is used to measure the response and recovery times of devices. The response and recovery times define the speed of the sensor in real-time optoelectronic applications. A notable point is that the HZFR showed the best response time of $\sim 7 \mathrm{~s}$ when compared to $\sim 12 \mathrm{~s}$ and $\sim 15 \mathrm{~s}$ in NRs and NFs, respectively. We certainly believe that the phenomenal $\sim 7 \mathrm{~s}$ is not only the best amongst the fellow nanostructures that are reported in this work, but it is the shortest response time ever to be reported via a ZnO-based passive sensor. Similarly, as soon as the UV was turned off, the HZFR device recovered very quickly when compared to the NR and NF sensors. Unlike the active devices, which provide power gain to the current response of the device, the presented devices are passive devices, that do not provide a power gain to the output current. Provided the passive nature of the devices, the response and recovery time behaviors of the devices are comparably better than those in recently published reports $[12,19]$.
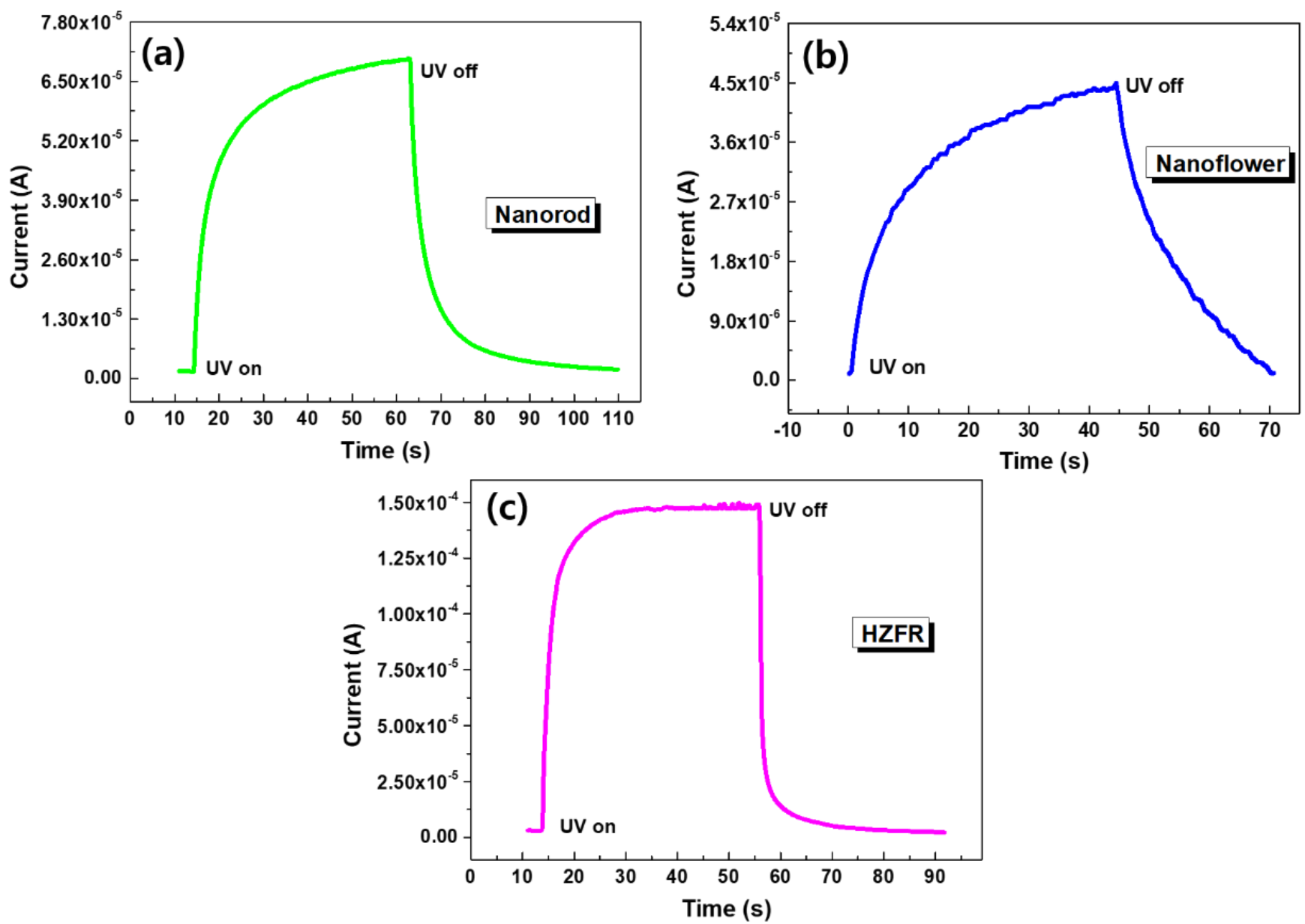

Figure 5. The impulse response is a function of time for (a) NRs; (b) NFs and (c) HZFR.

\subsection{Responsivity and Photoconductive Gain}

The equation that describes photoconductive gain $(G)$ is

$$
G=\frac{\tau}{t}
$$


where $(\tau)$ is the photo carriers' recombination time and $(t)$ is the impulse response time, previously found in Figure 6. Similarly, another equation for $G$ is

$$
G=R \cdot \frac{1.24}{\lambda} \cdot \frac{1}{\eta},
$$

where $R$ is the photodetector responsivity, $\eta$ is the quantum efficiency and $\lambda(368 \mathrm{~nm})$ is the UV source wavelength. Hence, the equation for $R$, drawn from Equation (4), is

$$
R=G \cdot \frac{\eta \cdot \lambda}{1.24}
$$

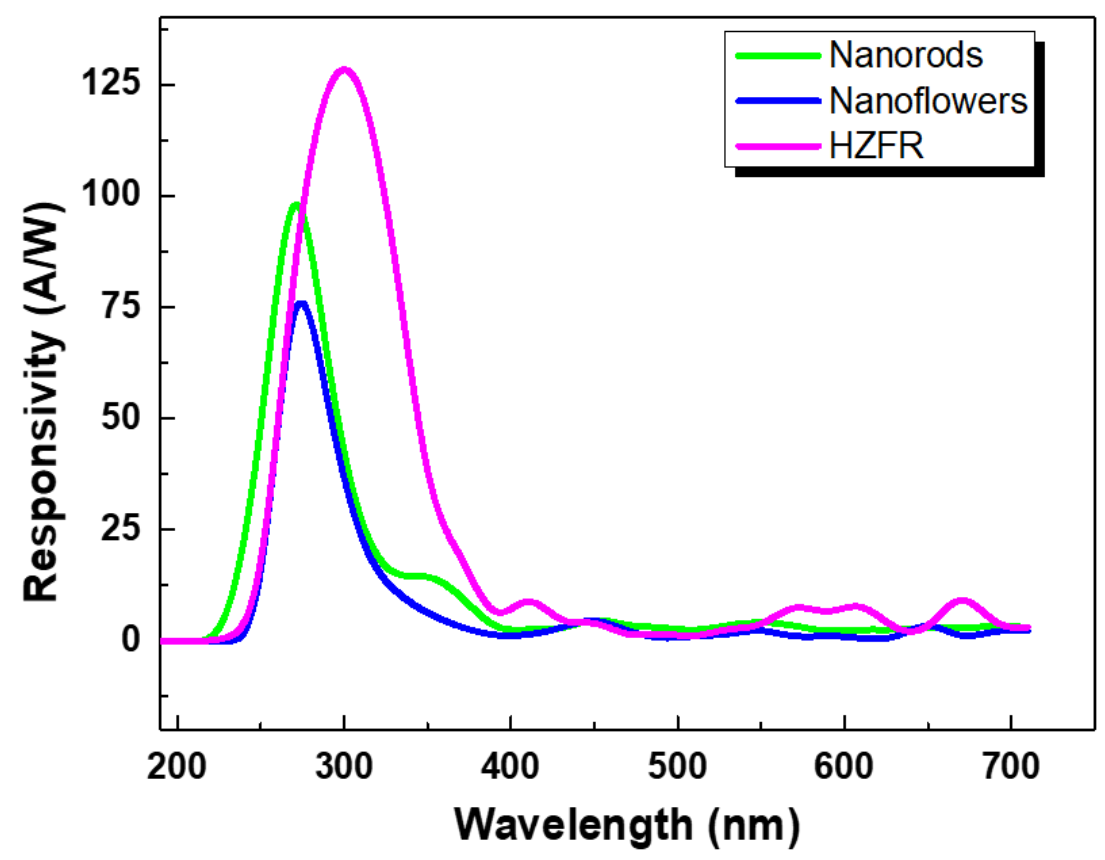

Figure 6. Sensors' responsivity as a function of wavelength.

It is evident from Equations (4) and (5) that $G$ and $R$ have a direct relationship. Hence, increasing the photoconductive gain directly improves the photodetector responsivity. Figure 6 shows the calculated $R$ for the three sensors containing NRs, NFs, and HZFR.

The highest $R$ of $126 \mathrm{~A} / \mathrm{W}$ was shown by the sensor that was fabricated with HZFR. The excessive light trapped by the HZFR was used to generate an enormous amount of e-h pairs in the channel. The excessive e-h pairs not only reduced the $t$, as reported in Figure 5 but also improved the $G$ of the sensor, as per Equation (3). Furthermore, the excessive e- $h$ pairs strengthened the avalanche process in the channel, which increased the sensor $G$ to the utmost degree. Consequently, the improved $G$ gave the best $R$-value for the sensors that were fabricated via HZFR, as reported in Figure 6. This high responsivity value (126 A/W) also demonstrates the fact that most of the photo-generated e-h pairs were collected at the reception terminals, which is highly expected of a good UV sensor. Without even using sophisticated external techniques, including the use of surfactants, antireflection coatings, Schottky barrier stimulation, and metal nanoparticle decoration, the presented results for HZFR are better than those in previously published reports [35-38], which is of great importance and success in the research and development on this important topic.

\subsection{Proposed UV Sensor Mechanism for HZFR}

Figure 7a-c shows the proposed UV sensor mechanism that was exploited in this study. They are the schematic representation of the sensing mechanism in NRs, NFs, and HZFR, and explain the reason why the HZFR sensor shows the best response. Figure 7a,b shows the patches and the spaces between the adjoining NRs and NFs, respectively, which 
led to the wasting of some UV light on the substrate. In this backdrop, we have three types of lights, namely, reflected light, absorbed light, and lost light. For the best UV sensor efficiency, the light absorption needs to be improved as much as possible. For this reason, researchers normally coat the $\mathrm{ZnO}$ layer with chemical absorbents, anti-reflection materials, and polymers [39]. Hence, we could not afford to lose any precious, non-reflected light to the substrate. Consequently, we introduced the HZFR, which not only trapped the majority of the absorbed light but also gave the best UV sensor results without using any foreign absorbents or material coatings, as reported in the above sections. Figure 7c shows how most of the light was absorbed and used for the e-h pairs generation by HZFR.

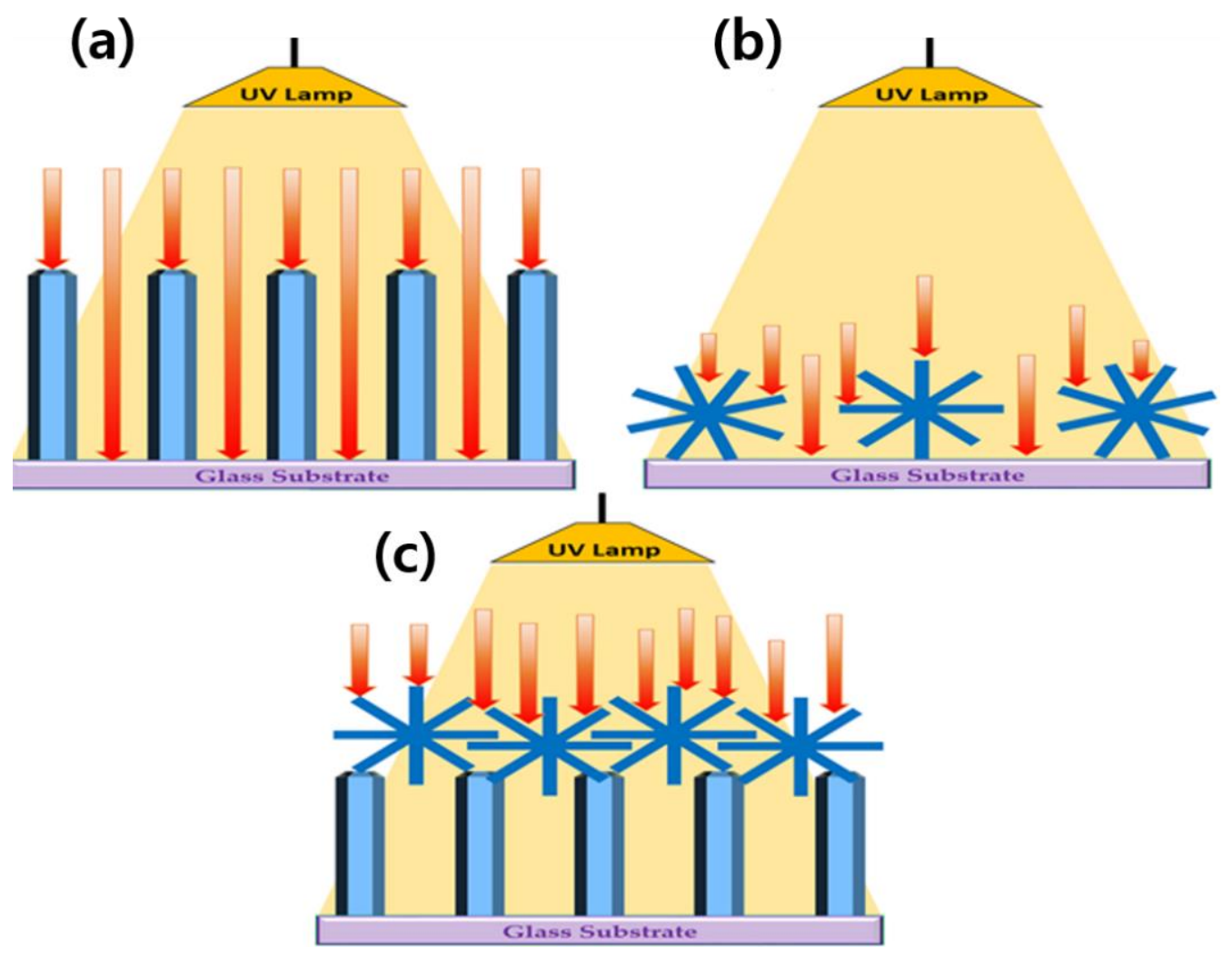

Figure 7. The UV sensor mechanism for (a) NRs and (b) NFs. (c) The proposed mechanism for HZFR.

Similar to the rest of the metal oxides, a Schottky barrier is formed at the $\mathrm{ZnO}$ interface via the oxygen chemisorption process, at ambient conditions [12,19]. This built-in barrier helps separate the UV-generated e-h pairs, where the generated holes are attracted towards the negatively charged surface and desorb the chemisorbed oxygen. Every desorbed oxygen molecule also adds an electron to the channel, increasing the overall electron density in the channel. The external bias voltage attracts the electrons to the metal contacts and leads to a certain current intensity. The overall UV sensor efficiency depends on the surface Schottky barrier height, the density of the chemisorbed oxygen, the density of the UV-generated e-h pairs, and the UV on-off current ratio. Various groups have proposed metal nanoparticles decoration on the metal oxide surfaces, to stimulate or strengthen the surface Schottky barriers $[40,41]$. Herein, the surface Schottky barriers were not stimulated via any external factors. Notwithstanding, the HZFR formed a more pronounced Schottky barrier at the NF-NR interface and facilitated a more pronounced special separation effect, for a better UV sensor efficiency. Furthermore, the charge transfer occurred at the NRNF interface via a phenomenon called a bridging circuit, which was first explored by Alenezi et al. in 2015 [42]. The top HZFR surface absorbed as much light as possible and generated a plethora of e-h pairs. The generated holes were used in surface oxygen desorption and the electrons were transferred to the buried NRs at the bridging tips. Hence, 
when compared to NRs and NFs, many electrons were collected at the bias terminals and the HZFR recorded the best UV sensor response, as was reported in earlier sections.

\section{Conclusions}

In this study, a novel HZFR was prepared for the first time and its UV sensor response was compared and contrasted with the traditional, and widely used, NRs and NFs. The active layers of the nanostructures were fabricated with the commercially benign and easygoing sol-gel MAG method. The physical, structural, optical, and electrical characteristics were tested via SEM, XRD, PL, and Keithley 4200A-SCS measurement setups, respectively. The focus of this study was neither stimulating the nanostructured surface Schottky barrier nor using any surfactants to enhance the UV light absorption. However, we paid attention to the basic ingredients of shaping the nanostructures' morphology with shapes that had never been previously used, to obtain the best results for UV sensors. The presented HZFR morphology exploits the benefits of both NFs and NRs by trapping as much light as possible for the generation of e-h pairs and transporting these smoothly to the receiver contacts. The top HZFR fingers trapped the whole UV light, and the converted e-h pairs were transmitted to the underlying NRs at the bridging interface tips of the flower and the buried rod structures in HZFR. The HZFR showed the best transient current response, with the least response and recovery times, and the highest UV on-off ratio. Similarly, it showed the best gain-induced responsivity among all the morphologies tested. It was concluded that the HZFR has emerged as a very effective morphology, for future applications in photodetectors, solar cells, gas sensors, and other optoelectronics applications. In the future, we would like to tune the crystal quality and the defect structures in the nascent morphologies for even better UV sensor results.

Author Contributions: Data curation, S.F.S. and A.M.A.-E.; formal analysis, A.M., M.U. and M.M.A.; funding acquisition, S.F.S. and A.M.A.-E.; investigation, S.F.S.; methodology, A.M. and M.F.I.; project administration, S.F.S.; resources, A.M.A.-E. and S.F.S.; software, A.M.T. and S.U.E.; supervision, A.M.A.-E., S.F.S. and A.M.A.-E.; writing—original draft, A.M.A.-E.; writing—review \& editing, S.F.S., A.M.T. and A.M.A.-E. All authors have read and agreed to the published version of the manuscript.

Funding: This research was funded by the Researchers supporting project number (RSP-2021/370), King Saud University, Riyadh, Saudi Arabia.

Institutional Review Board Statement: Not applicable.

Informed Consent Statement: Not applicable.

Data Availability Statement: Data is contained within the article.

Acknowledgments: The authors would like to extend their sincere appreciation to the Researchers supporting project number (RSP-2021/370), King Saud University, Riyadh, Saudi Arabia for funding this research.

Conflicts of Interest: The authors declare no conflict of interest.

\section{References}

1. Amin, M.; Shah, N.A.; Bhatti, A.S. Development of highly sensitive UV sensor using morphology tuned ZnO nanostructures. Appl. Phys. A 2015, 118, 595-603. [CrossRef]

2. Zhang, X.; Han, X.; Su, J.; Zhang, Q.; Gao, Y. Well vertically aligned ZnO nanowire arrays with an ultra-fast recovery time for UV photodetector. Appl. Phys A 2012, 107, 255-260. [CrossRef]

3. Caruge, J.M.; Halpert, J.E.; Wood, V.; Bulovic, V.; Bawendi, M.G. Colloidal quantum-dot light-emitting diodes with metal-oxide charge transport layers. Nat. Photonics 2008, 2, 247-250. [CrossRef]

4. Anikeeva, P.O.; Halpert, J.E.; Bawendi, M.G.; Bulovic, V. Electroluminescence from a mixed red-green-blue colloidal quantum dot monolayer. Nano Lett. 2007, 7, 2196-2200. [CrossRef]

5. Rana, A.S.; Kim, H.S. $\mathrm{NH}_{4} \mathrm{OH}$ Treatment for an optimum morphological trade-off to hydrothermal Ga-doped n-ZnO/p-Si heterostructure characteristics. Materials 2018, 11, 37. [CrossRef]

6. Li, H.; Zhang, X.; Liu, N.; Ding, L.; Tao, J.; Wang, S.; Su, J.; Li, L.; Gao, Y. Enhanced photo-response properties of a single ZnO microwire photodetector by coupling effect between localized Schottky barriers and piezoelectric potential. Opt. Express 2015, 23, 21204-21212. [CrossRef] 
7. Rana, A.S.; Kang, M.; Jeong, E.S.; Kim, H.S. Transition between ZnO Nanorods and ZnO Nanotubes with Their Antithetical Properties. J. Nanosci. Nanotechnol. 2016, 16, 10772-10776. [CrossRef]

8. Liu, Y.; Zhang, X.; Su, J.; Li, H.; Zhang, Q.; Gao, Y. Ag nanoparticles@ZnO nanowire composite arrays: An absorption enhanced UV photodetector. Opt. Express 2014, 22, 30148-30155. [CrossRef]

9. Kim, H.; Kim, W.; Park, J.; Lim, M.; Lee, R.; Cho, S.J.; Kumaresan, Y.; Oh, M.K.; Jun, G.Y. Surface conversion of ZnO nanorods to ZIF-8 to suppress surface defects for a visible-blind UV photodetector. Nanoscale 2018, 10, 21168-21177. [CrossRef]

10. Grottrup, J.; Postica, V.; Smazna, D.; Hoppe, M.; Kaidas, V.; Mishra, Y.K.; Lupan, O.; Adelunga, R. UV detection properties of hybrid ZnO tetrapod 3-D networks. Vacuum 2017, 146, 492-500. [CrossRef]

11. Weber, M.; Kim, J.Y.; Lee, J.H.; Kim, J.H.; Iatsunskyi, I.; Coy, E.; Miele, P.; Bechelany, M.; Kim, S.S. Highly efficient hydrogen sensors based on Pd nanoparticles supported on boron nitride coated ZnO nanowires. J. Mater. Chem. A 2019, 7, 8107-8116. [CrossRef]

12. Rana, A.S.; Lee, J.Y.; Hong, Y.P.; Kim, H.S. Transient Current Response for ZnO Nanorod-Based Doubly Transparent UV Sensor Fabricated on Flexible Substrate. Phys. Status Solidi RRL 2018, 12, 1800001. [CrossRef]

13. Yang, Z.; Wang, M.; Zhao, Q.; Qiu, H.; Li, J.; Li, X.; Shao, J. Dielectrophoretic-Assembled Single and Parallel-Aligned Ag Nanowire-ZnO-Branched Nanorod Heteronanowire Ultraviolet Photodetectors. ACS Appl. Mater. Interfaces 2017, 9, 22837-22845. [CrossRef]

14. Boscarino, S.; Filice, S.; Sciuto, A.; Libertino, S.; Scuderi, M.; Galati, C.; Scalese, S. Investigation of ZnO-decorated CNTs for UV light detection applications. Nanomaterials 2019, 9, 1099. [CrossRef]

15. Singh, S.; Kumar, Y.; Kumar, H.; Vyas, S.; Periasamy, C.; Chakrabarti, P.; Park, S.H. A study of hydrothermally grown ZnO nanorod-based metal-semiconductor-metal UV detectors on glass substrates. Nanomater. Nanotechnol. 2017, 7, 1847980417702144. [CrossRef]

16. Tahira, A.; Mazzaro, R.; Rigoni, F.; Nafady, A.; Shaikh, S.F.; Al-Othman, A.A.; Alshgari, R.A.; Ibupoto, Z.H. A simple and efficient visible light photodetector based on $\mathrm{Co}_{3} \mathrm{O}_{4} /$ Zno composite. Opt. Quantum Electron. 2021, 53, 1-9. [CrossRef]

17. Luo, L.; Zhang, Y.; Mao, S.S.; Lin, L. Fabrication and characterization of ZnO nanowires based UV photodiodes. Sens. Actuators A-Phys. 2006, 127, 201-206. [CrossRef]

18. Zhou, J.; Gu, Y.; Hu, Y.; Mai, W.; Yeh, P.H.; Bao, G.; Sood, A.K.; Polla, D.L.; Wang, Z.L. Gigantic enhancement in response and reset time of ZnO UV nanosensor by utilizing Schottky contact and surface functionalization. Appl. Phys. Lett. 2009, 94, 191103. [CrossRef]

19. Shaikh, S.F.; Al-Enizi, A.M.; Agyeman, D.A.; Ghani, F.; Nah, I.W.; Shahid, A. Intrinsic control in defects density for improved ZnO nanorod-based UV sensor performance. Nanomaterials 2020, 10, 142.

20. Yang, P.; Yan, H.; Mao, S.; Russo, R.; Johnson, J.; Saykally, R.; Morris, N.; Pham, J.; He, R.; Choi, H.J. Controlled growth of ZnO nanowires and their optical properties. Adv. Funct. Mater. 2002, 12, 323-331. [CrossRef]

21. Park, W.I.; Yi, G.C.; Kim, M.; Pennycook, S.J. ZnO nanoneedles grown vertically on Si substrates by non-catalytic vapor-phase epitaxy. Adv. Mater. 2002, 14, 1841-1843. [CrossRef]

22. Sun, Y.; Fuge, G.M.; Ashfold, M.N. Growth of aligned ZnO nanorod arrays by catalyst-free pulsed laser deposition methods. Chem. Phys. Lett. 2004, 396, 21-26. [CrossRef]

23. Yao, B.D.; Chan, Y.F.; Wang, N. Formation of ZnO nanostructures by a simple way of thermal evaporation. Appl. Phys. Lett. 2002, 81,757-759. [CrossRef]

24. Rana, A.S.; Ko, K.; Hong, S.; Kang, M.; Kim, H.S. Fabrication and characterization of ZnO nanorods on multiple substrates. J. Nanosci. Nanotechnol. 2015, 15, 8375-8380. [CrossRef]

25. Rana, A.S.; Kang, M.; Kim, H.S. Microwave-assisted facile and ultrafast growth of ZnO nanostructures and proposition of alternative microwave-assisted methods to address growth stoppage. Sci. Rep. 2016, 6, 24870. [CrossRef]

26. Wojnarowicz, J.; Chudoba, T.; Lojkowski, W. A review of microwave synthesis of zinc oxide nanomaterials: Reactants, process parameters and morphologies. Nanomaterials 2020, 10, 1086. [CrossRef]

27. Chang, S.B.; Chae, H.U.; Kim, H.S. Structural, optical, electrical and morphological properties of different concentration sol-gel $\mathrm{ZnO}$ seeds and consanguineous $\mathrm{ZnO}$ nanostructured growth dependence on seeds. J. Alloys Comp. 2017, 729, 571-582.

28. Luo, J.; Ma, S.Y.; Sun, A.M.; Cheng, L.; Yang, G.J.; Wang, T.; Li, W.Q.; Li, X.B.; Mao, Y.Z.; Gz, D.J. Ethanol sensing enhancement by optimizing ZnO nanostructure: From 1D nanorods to 3D nanoflower. Mater. Lett. 2014, 137, 17-20. [CrossRef]

29. Sahdan, M.Z.; Mamat, M.H.; Salina, M.; Khusaimi, Z.; Noor, U.M.; Rusop, M. Heat treatment effects on the surface morphology and optical properties of ZnO nanostructures. Phys. Status Solidi C 2010, 7, 2286-2289. [CrossRef]

30. Keskenler, E.F.; Tomakin, M.; Dogan, S.; Turgut, G.; Aydın, S.; Duman, S.; Gurbulak, B. Growth and characterization of $\mathrm{Ag} / \mathrm{n}-\mathrm{ZnO} / \mathrm{p}-\mathrm{Si} / \mathrm{Al}$ heterojunction diode by solegel spin technique. J. Alloys Compd. 2013, 550, 129-132. [CrossRef]

31. Rana, A.S.; Lee, J.Y.; Shahid, A.; Kim, H.S. Growth method-dependent and defect density-oriented structural, optical, conductive, and physical properties of solution-grown ZnO nanostructures. Nanomaterials 2017, 7, 266. [CrossRef]

32. Janotti, A.; Van de Walle, C.G. Native point defects in ZnO. Phys. Rev. B 2007, 76, 165202. [CrossRef]

33. Reynolds, D.C.; Look, D.C.; Jogai, B. Combined effects of screening and band gap renormalization on the energy of optical transitions in ZnO and GaN. J. Appl. Phys. 2000, 88, 5760-5763. [CrossRef]

34. Fair, R.B. The effect of strain-induced band-gap narrowing on high concentration phosphorus diffusion in silicon. J. Appl. Phys. 1979, 50, 860-868. [CrossRef] 
35. Rodwihok, C.; Choopun, S.; Ruankham, P.; Gardchareon, A.; Phadungdhitidhada, S.; Wongratanaphisan, D. UV sensing properties of ZnO nanowires/nanorods. Appl. Surf. Sci. 2019, 477, 159-165. [CrossRef]

36. Eom, T.H.; Han, J.I. Single fiber UV detector based on hydrothermally synthesized ZnO nanorods for wearable computing devices. Appl. Surf. Sci. 2018, 428, 233-241. [CrossRef]

37. Makhlouf, H.; Karam, C.; Lamouchi, A.; Tingry, S.; Miele, P.; Habchi, R.; Chtourou, R.; Bechelany, M. Analysis of ultraviolet photo-response of $\mathrm{ZnO}$ nanostructures prepared by electrodeposition and atomic layer deposition. Appl. Surf. Sci. 2018, 444, 253-259. [CrossRef]

38. Yu, J.; Shan, C.X.; Qiao, Q.; Xie, X.H.; Wang, S.P.; Zhang, Z.Z.; Shen, D.Z. Enhanced responsivity of photodetectors realized via impact ionization. Sensors 2012, 12, 1280-1287. [CrossRef]

39. Zhang, H.; Hu, Y.; Wang, Z.; Fang, Z.; Peng, L.M. Performance boosting of flexible ZnO UV sensors with rational designed absorbing antireflection layer and humectant encapsulation. ACS Appl. Mater. Interf. 2016, 8, 381-389. [CrossRef]

40. Wang, X.; Liu, K.; Chen, X.; Li, B.; Jiang, M.; Zhang, Z.; Zhao, H.; Shen, D. Highly wavelength-selective enhancement of responsivity in Ag nanoparticle-modified ZnO UV photodetector. ACS Appl. Mater. Interf. 2017, 9, 5574-5579. [CrossRef]

41. Li, Y.; Gong, J.; He, G.; Deng, Y. Enhancement of photoresponse and UV-assisted gas sensing with Au decorated ZnO nanofibers. Mater. Chem. Phys. 2012, 134, 1172-1178. [CrossRef]

42. Alenezi, M.R.; Henley, S.J.; Silva, S.R.P. On-chip fabrication of high performance nanostructured ZnO UV detectors. Sci. Rep. 2015, 5, 1-9. [CrossRef] 